\title{
Xp11.2 translocation renal cell carcinoma with multiple bone metastases: A case report
}

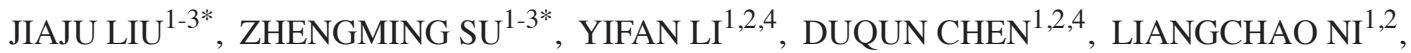 \\ XIANGMING MAO ${ }^{1,2}$, SHANGQI YANG ${ }^{1,2}$ and YONGQING LAI ${ }^{1,2}$ \\ ${ }^{1}$ Department of Urology, Peking University Shenzhen Hospital; \\ ${ }^{2}$ The Guangdong and Shenzhen Key Laboratory of Male Reproductive Medicine and Genetics, \\ Peking University Shenzhen Hospital, Institute of Urology of Shenzhen PKU-HKUST Medical Center, Shenzhen, \\ Guangdong 518036; ${ }^{3}$ Department of Urology, Shantou University Medical College, Shantou, Guangdong 515041; \\ ${ }^{4}$ Department of Urology, Anhui Medical University, Hefei, Anhui 230032, P.R. China
}

Received November 25, 2014; Accepted December 18, 2015

DOI: $10.3892 / \mathrm{ol} .2016 .4211$

\begin{abstract}
Xp11.2 translocation/transcription factor enhancer 3 (TFE3) fusion gene associated with renal cell carcinoma (Xp11.2 translocation RCC) is rare and occurs predominantly in children and adolescents. The current study reports the case of a 14-year-old male with Xp11.2 translocation RCC, who presented with chest pain that had persisted for 1 month. A solid neoplasm was located in the left kidney of the patient. Contrast-enhanced computed tomography revealed the presence of a solid mass in the kidney, with uneven enhancement. Destruction of multiple bones was also observed. The patient was treated with a radical nephrectomy. The pathological examination of the tumor revealed that the tumor cells contained an eosinophilic cytoplasm in the renal interstitial tissue. Immunohistochemistry revealed that the tumor cells expressed P504S, cluster of differentiation 10, pan-cytokeratin, vimentin and TFE3. In conclusion, Xp11.2 translocation RCC is a rare type of kidney cancer. Diagnosing this disease prior to surgery is challenging, and providing a definite diagnosis requires histopathological and immunohistochemical examination, while genetic analysis may also be required.
\end{abstract}

\section{Introduction}

The most common kidney cancer is renal cell carcinoma (RCC), which accounts for 2-3\% of all types of cancer worldwide (1). The prognosis of RCC varies according to the stage and histological grade of the tumor, and with genetic

Correspondence to: Professor Yongqing Lai, Department of Urology, Peking University Shenzhen Hospital, 1120 Lianhua Road, Shenzhen, Guangdong 518036, P.R. China

E-mail: yqlord@163.com

*Contributed equally

Key words: Xp11.2, renal cell carcinoma, metastases, TFE3 profiling and technological improvements, the classification of RCC has expanded. In the 2004 World Health Organization classification of renal tumors, RCC associated with Xp11.2 translocation/transcription factor enhancer 3 (TFE3) fusion gene (Xp11.2 translocation RCC) is described as a distinct type of RCC (2). Xp11.2 translocation RCC is diagnosed primarily in children, where it accounts for $30 \%$ of pediatric RCC cases; however, RCC translocations have recently been observed in adults, who have a poorer prognosis compared with children (3). This type of RCC is characterized by a range of chromosome translocations, each of which consists of a breakpoint at Xp11.2 and a fusion involving the TFE3 gene. The current study describes the case of a 14-year-old boy who presented with chest pain that had persisted for 1 month. A kidney neoplasm was incidentally located using computed tomography (CT). The type of RCC may usually be diagnosed according to the pathology of the tumor, and no unique treatment of RCC, particularly the Xp11.2 translocation, exists. Therefore, surgical treatment was used in the present study, based on the characteristics of the tumor, including its size and adhesion with the surrounding tissue. The present study also reviewed the literature concerning Xp11.2 translocation $\mathrm{RCC}$, in order to improve the diagnosis and treatment of this rare disease.

\section{Case report}

A previously healthy 14-year-old male presented to Department of Urology, Peking University Shenzhen Hospital, (Shenzhen, China) on July 13, 2014, with chest pain that had persisted for 1 month. The patient had no significant medical history, no family history of cancer and did not smoke or drink alcohol. A physical examination was negative, but a urine occult blood test was positive. An ultrasound revealed the presence of a solid mass in the left kidney, with a mean diameter of $6.0 \times 5.4 \mathrm{~cm}$. Abdominal contrast-enhanced CT (Sensation 16 MDCT scanner; Siemens Healthcare, Erlangen, Germany) demonstrated the presence of a solid mass in the left kidney, with uneven enhancement (Fig. 1A and B). In addition, there was destruction of multiple bones, including the 
A



C

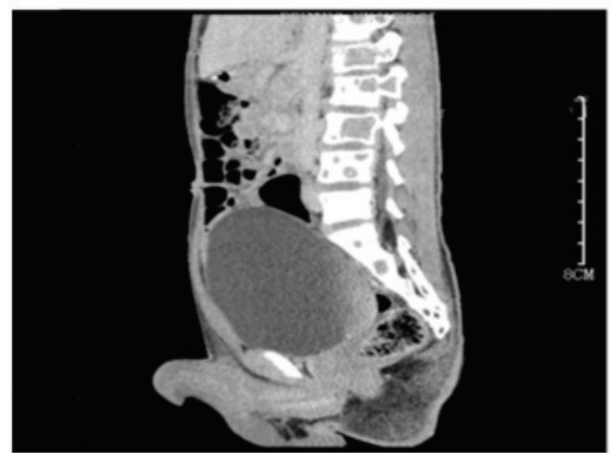

B

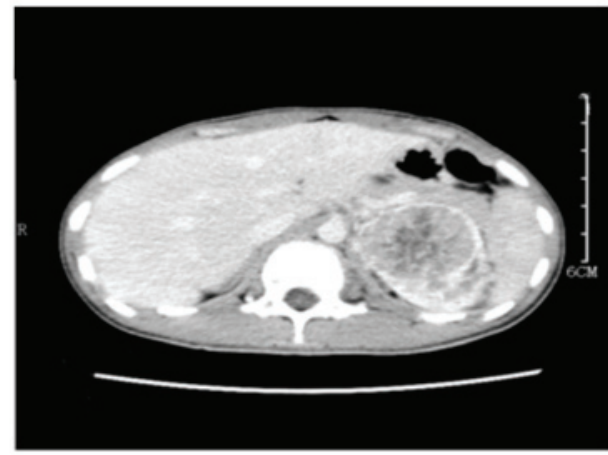

D

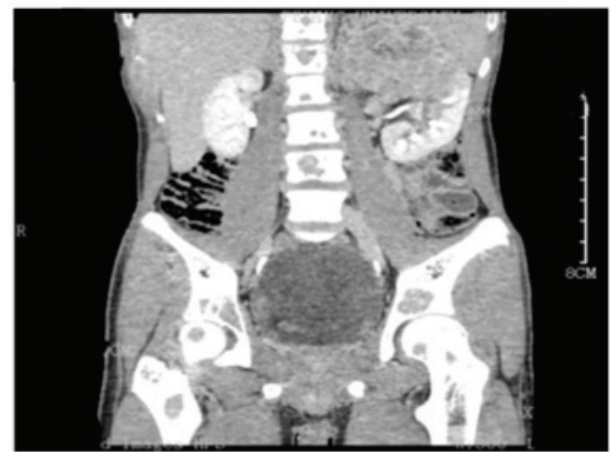

Figure 1. (A and B) CT axial sections revealing the presence of an ill-defined neoplasm with a thick wall, measuring $8 \mathrm{~cm}$ in diameter, on the upper pole of the left kidney. CT (C) sagittal and (D) coronal section demonstrating the presence of multiple bone destruction, including the vertebrae, pelvis and bilateral femoral head. CT, computed tomography.



Figure 2. Histological findings of Xp11.2 translocation/transcription factor enhancer 3 fusion gene associated with renal cell carcinoma. The tumor was composed of clear cells with voluminous eosinophilic cytoplasm and distinct cell borders exhibiting typical papillary architecture (hematoxylin and eosin; magnification, $\mathrm{x} 100$ ).



Figure 3. Histological findings of Xp11.2 translocation/transcription factor enhancer 3 fusion gene associated with renal cell carcinoma, showing clear psammoma bodies (hematoxylin and eosin; magnification, x100).

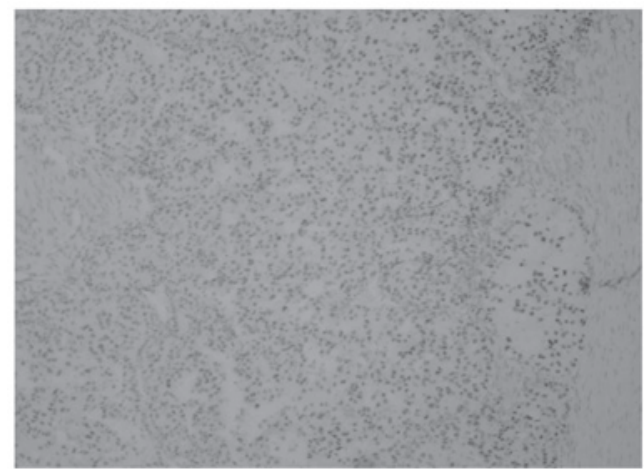

Figure 4. Immunohistochemical findings of Xp11.2 translocation/transcription factor enhancer 3 fusion gene associated with renal cell carcinoma, showing the tumor clearly and diffusely expressing nuclear transcription factor for immunoglobulin heavy-chain enhancer 3 (hematoxylin and eosin; magnification, $\mathrm{x} 400$ ).

vertebrae, pelvis and bilateral femoral head (Fig. 1C and D). A left radical nephrectomy and partial ureterectomy were performed.

Intraoperatively, the left kidney was clearly enlarged and severe adhesions were observed. The surrounding fat tissues were easily identified. Macroscopically, the ill-defined tumor was located in the upper pole of the resected kidney and measured $7.0 \times 7.0 \times 4.5 \mathrm{~cm}$ in size. There were several sporadic tumor nodules, with a mean diameter of $3.0 \mathrm{~mm}$, which were all well-circumscribed. The gross appearance of the tumor was yellow, hemorrhagic and necrotic, which is similar to the appearance of conventional RCC (4).

The formalin-fixed, paraffin-embedded tissue ( $4 \mu \mathrm{m}$ pathological section) was used for immunohistochemical 
analysis with the primary antibody TFE3 (clone P-16; goat anti-human polyclonal antibody; cat. no. sc-5958; Santa Cruz Biotechnology, Santa Cruz, CA, USA), according to the manufacturer's overnight incubation methodology. The tumor was stained with hematoxylin and eosin. Microscopically, the tumor was composed of cells arranged in primarily papillary patterns, with abundant clear to eosinophilic cytoplasm and prominent nucleoli (Fig. 2). Psammoma bodies were frequently observed (Fig. 3). Hemorrhage, necrosis and multifocal calcifications were also clearly observed in the tumor. None of the hilar lymph nodes exhibited neoplastic infiltration.

Immunohistochemistry demonstrated that the neoplastic cells clearly and diffusely expressed TFE3 (Fig. 4) and cluster of differentiation 10, and focally expressed P504S, pan-cytokeratin, vimentin, carbonic anhydrase IX and kidney-specific cadherin. The patient was diagnosed with Xp11.2 translocation RCC and multiple bone metastases.

Following the surgery, the patient was transfered to another hospital to receive further treatment, the detail of which is unknown. However, the patient's family informed the Department of Urology, Peking University Shenzhen Hospital, that the patient succumbed 6 months later.

\section{Discussion}

Xp11.2 translocation RCC is characterized by several translocations that involve the TFE3 gene, which is located on chromosome Xp11.2, and leads to genic fusions. There are 5 gene partners of TFE3, including papillary renal cell carcinoma (translocation-associated) (PRCC), alveolar soft part sarcoma chromosome region, candidate 1 (ASPL), splicing factor proline/glutamine-rich (PSF), non-POU domain containing, octamer-binding and clathrin, heavy chain, which are located on 1q21, 17q25, 1p34, Xq12 and 17q23, respectively. The most common translocations are $\mathrm{t}(\mathrm{X} ; 17)$ (p11.2;q25), t(X;1)(p11.2;p34) and $\mathrm{t}(\mathrm{X} ; 1)(\mathrm{p} 11.2 ; \mathrm{q} 21)$, which result in gene fusions of TFE3 with ASPL, PSF and PRCC, respectively $(3,5,6)$.

Macroscopically, Xp11.2 translocation RCC is heterogeneous. The tumors are usually tan-yellow or grey-white, necrotic and hemorrhagic, which is similar to the appearance of classic RCC. The most distinctive histopathological appearance is the papillary and nested architecture that is composed of a voluminous clear to eosinophilic cytoplasm, prominent nucleoli, vesicular chromatin, discrete cell borders, scattered hyaline nodules and psammoma bodies (7-9). Tumors of the PRCC-TFE3 fusion gene are usually composed of intermediate-sized cells and exhibit few psammoma bodies. By contrast, tumors of the ASPL-TFE3 fusion gene exhibit voluminous cells that are often dyscohesive and have an alveolar and pseudopapillary architecture, hyaline nodules and extensive psammoma bodies, which is similar to the characteristics observed in the present case $(6-8,10)$.

Diagnosing Xp11.2 translocation RCC from other types of RCC that are often observed in patients, such as clear cell RCC and papillary RCC, is relatively uncomplicated due to several important defining characteristics. First, the majority of Xp11.2 translocation RCC cases occur in children. While RCC accounts for $5 \%$ of pediatric renal tumors, $\mathrm{Xp11.2}$ translocation
RCC may account for $>33 \%$. Therefore, the presence of RCC in a child or young adult may indicate Xp11.2 translocation RCC (11). Although Xp11.2 translocation RCC has been recently reported in adults, it remains uncommon, which may lead to these tumors being misdiagnosed as clear cell or papillary RCC. Second, the histological characteristics observed with Xp11.2 translocation RCC may differentiate the tumor from non-classical RCC, including tumor cells with a voluminous cytoplasm in a papillary arrangement. Third, using anti-TFE3 antibodies in immunohistochemistry may lead to the correct diagnosis; XP11.5 translocation RCC is confirmed by the detection of chromosome translocation involving the TFE3 gene at Xp11.2 using various methods.

In the current case, the patient possessed with an aggressive tumor. The patient originally presented with a long-term history of chest pain, and a solid mass was detected in the kidney, with multiple bone destruction, by CT. Therefore, we hypothesize that the tumors developed when the patient was younger or in childhood, and were not observed until the masses become large and were at an advanced stage. The treatment of the patient was primarily surgical. Chemotherapy should be a treatment of choice; however, its affect in advanced-stage Xp11.2 translocation RCC is poor. In addition, Kmetec and Jeruc (9) reported the case of a patient with recurrent tumors following surgery for Xp11.2 translocation RCC. The patient was administered with sunitinib and everolimus (a mechanistic target of rapamycin inhibitor), but no effective response resulted. Furthermore, certain studies have demonstrated that prior administration of chemotherapy may be a risk factor for developing translocation carcinomas (12). Therefore, the advantages and disadvantages of chemotherapy in patients with Xp11.2 translocation RCC should be evaluated prior to administration.

To relieve the severe clinical outcome that is associated with Xp11.2 RCC, early detection, accurate diagnosis and close follow-up are required. Prior to the development of radical nephrectomy there was no effective surveillance method for Xp11.2 translocation RCC. Klaassen et al (13) recommended aggressive follow-up with regular physical examination, laboratory tests, and chest and abdominal $\mathrm{CT}$, for up to 10 years duration. Furthermore, since Xp11.2 translocation RCC is often diagnosed in young adults, the study proposed lifelong follow-up with a yearly physical examination, laboratory tests, and chest and abdominal imaging subsequent to the completion of the 10-year follow-up.

$\mathrm{Xp} 11.2$ translocation RCC is a newly described, but rarely encountered subtype of RCC. It normally presents with specific morphogenetic characteristics and biological behavior. The detection of TFE3 expression using immunohistochemistry appears to be an easy and useful technique to identify this type of tumor. However, genetic analysis is required to elucidate the type of gene fusion.

\section{Acknowledgements}

The present study was supported by the National Natural Science Foundation of China (grant no. 81101922) and the Science and Technology Development Fund Project of Shenzhen (grant nos. JCYJ20130402114702124 and JCYJ20150403091443329). 


\section{References}

1. Ferlay J, Soerjomataram I, Dikshit R, Eser S, Mathers C, Rebelo M, Parkin DM, Forman D and Bray F: Cancer incidence and mortality worldwide: Sources, methods and major patterns in GLOBOCAN 2012. Int J Cancer 136: E359-E386, 2015.

2. Lopez-Beltran A, Scarpelli M, Montironi R and Kirkali Z: 2004 WHO classification of the renal tumors of the adults. Eur Urol 49: 798-805, 2006.

3. Argani P and Ladanyi M: Translocation carcinomas of the kidney. Clin Lab Med 25: 363-378, 2005.

4. Ross H, Martignoni G and Argani P: Renal cell carcinoma with clear cell and papillary features. Arch Pathol Lab Med 136: 391-399, 2012.

5. Armah HB and Parwani AV: Xp11.2 translocation renal cell carcinoma. Arch Pathol Lab Med 134: 124-129, 2010.

6. Argani P and Ladanyi M: Renal carcinomas associated with Xp11.2 translocations/TFE3 gene fusions. In: World Health Organization Classification of Tumours. Pathology and Genetics of Tumours of the Urinary System and Male Genital Organs. Eble JN, Sauter G, Epstein JI and Sesterhenn IA (eds). IARC Press, Lyon, pp37-38, 2004.

7. ArganiP,AntonescuCR,IlleiPB,LuiMY,Timmons CF,NewburyR, Reuter VE, Garvin AJ, Perez-Atayde AR, Fletcher JA, et al Primary renal neoplasms with the ASPL-TFE3 gene fusion of alveolar soft part sarcoma: A distinctive tumor entity previously included among renal cell carcinomas of children and adolescents Am J Pathol 159: 179-192, 2001.
8. Argani P, Lal P, Hutchinson B, Lui MY, Reuter VE and Ladanyi M: Aberrant nuclear immunoreactivity for TFE3 in neoplasms with TFE3 gene fusions: A sensitive and specific immunohistochemical assay. Am J Surg Pathol 27: 750-761, 2003.

9. Kmetec A and Jeruc J: Xp 11.2 translocation renal carcinoma in young adults; recently classified distinct subtype. Radiol Oncol 48: 197-202, 2014.

10. Argani P, Antonescu CR, Couturier J, Fournet JC, Sciot R, Debiec-Rychter M, Hutchinson B, Reuter VE, Boccon-Gibod L, Timmons C, et al: PRCC-TFE3 renal carcinomas: Morphologic, immunohistochemical, ultrastructural and molecular analysis of an entity associated with the $\mathrm{t}(\mathrm{X} ; 1)(\mathrm{p} 11.2 ; \mathrm{q} 21)$. Am J Surg Pathol 26: 1553-1566, 2002.

11. Schinstine M, Filie AC, Torres-Cabala C, Abati A, Linehan WM and Merino M: Fine-needle aspiration of a Xp11.2 translocation/TFE3 fusion renal cell carcinoma metastatic to the lung: Report of a case and review of the literature. Diagn Cytopathol 34: 751-756, 2006

12. Argani P, Laé M, Ballard ET, Amin M, Manivel C, Hutchinson B, Reuter VE and Ladanyi M: Translocation carcinomas of the kidney after chemotherapy in childhood. J Clin Oncol 24: 1529-1534, 2006

13. Klaassen Z, Tatem A, Burnette JO, Donohoe JM and Terris MK: Adult Xp11 translocation associated renal cell carcinoma: Time to recognize. Urology 80: 965-968, 2012. 